\title{
Prevalence and resistance pattern of Moraxella catarhalis in community-acquired lower respiratory tract infections
}

This article was published in the following Dove Press journal:

Infection and Drug Resistance

31 July 2015

Number of times this article has been viewed

\section{Safia Bader Uddin Shaikh \\ Zafar Ahmed \\ Syed Ali Arsalan \\ Sana Shafiq}

Department of Pulmonology, Liaquat National Hospital, Karachi, Pakistan

Correspondence:

Dr Safia Bader Uddin Shaikh

Apt 324, 7225 Fair Oaks Avenue, Dallas,

TX 7523I, USA

Email safia.b.shaikh@gmail.com
Introduction: Moraxella catarrhalis previously considered as commensal of upper respiratory tract has gained importance as a pathogen responsible for respiratory tract infections. Its beta-lactamase-producing ability draws even more attention toward its varying patterns of resistance.

Methods: This was an observational study conducted to evaluate the prevalence and resistance pattern of $M$. catarrhalis. Patients aged 20-80 years admitted in the Department of Chest Medicine of Liaquat National Hospital from March 2012 to December 2012 were included in the study. Respiratory samples of sputum, tracheal secretions, and bronchoalveolar lavage were included, and their cultures were followed.

Results: Out of 110 respiratory samples, 22 showed positive cultures for M. catarrhalis in which 14 were males and eight were females. Ten samples out of 22 showed resistance to clarithromycin, and 13 samples out of 22 displayed resistance to erythromycin, whereas 13 showed resistance to levofloxacin. Hence, $45 \%$ of the cultures showed resistance to macrolides so far and $59 \%$ showed resistance to quinolones.

Conclusion: Our study shows that in our environment, M. catarrhalis may be resistant to macrolides and quinolones; hence, these should not be recommended as an alternative treatment in community-acquired lower respiratory tract infections caused by $M$. catarrhalis. However, a study of larger sample size should be conducted to determine if the recommendations are required to be changed.

Keywords: community-acquired lower respiratory tract infections or pneumonia, M. catarrhalis, antibiotic resistance, gram-negative diplococcic, Pakistan

\section{Introduction}

Moraxella catarrhalis is a gram-negative diplococcus, formerly known as Neisseria catarrhalis or Branhamella catarrhalis, that is found in the human upper respiratory tract as normal flora and was considered to occasionally cause infections. ${ }^{1}$ Currently, $M$. catarrhalis is a recognized pathogen of upper and lower respiratory tract infections. ${ }^{2}$ It has been found as the causative agent in infections, such as empyema, endocarditis, otitis media, and pneumonia, both in children and adults. ${ }^{3,4}$ The beta-lactamase-producing M. catarrhalis not reported before 1976 is the significant cause of varying patterns of resistance. ${ }^{5}$ The increase in occurrence of beta-lactamase strains can be regarded as the fastest dissemination of beta-lactamases within a bacterial species. ${ }^{6} \mathrm{M}$. catarrhalis has particularly become an important pathogen in patients with immunocompromised status and in patients with chronic pulmonary diseases. ${ }^{7}$ The aim of this study is to determine the prevalence of $M$. catarrhalis in our setup in patients presenting with the diagnosis 
of community-acquired lower respiratory tract infection and to determine the pattern of antibiotic sensitivity and resistance in these cases.

\section{Method}

This was an observational study conducted in the Department of Chest Medicine of Liaquat National Hospital and Medical College from March 2012 to December 2012. Approval was obtained from the hospital administration to conduct this study. Patients aged between 20 years and 80 years with the diagnosis of community-acquired lower respiratory tract infection were included in the study. Microsoft Excel 2007 was used to analyze the data.

The inclusion criteria were patients aged between 20 years and 80 years; those with productive cough; those with positive infective markers such as high white blood cell count, fever, or high C-reactive protein (CRP); and those with cultures positive for $M$. catarrhalis in their respiratory sample collected on admission. The results reviewed were from inpatient records only. The exclusion criteria were patients referred from periphery hospital where they have spent 48 hours or more with a suspicion of hospital-acquired lower respiratory tract infection. It was mandatory to acquire at least three consecutive samples of sputum to rule out the commensal organisms. Patients with positive culture in less than two samples or with cultures positive for organisms other than or in addition to $M$. catarrhalis were excluded from the study.

A total of 36 sputum samples were evaluated, three from each patient, and after determining the true pathogen, only one sample from each patient was considered for the study giving us a total of twelve sputum samples. In addition three samples of bronchoalveolar lavage and seven samples of tracheal secretions were also evaluated. Purulent parts of the samples were examined and stained with gram stain. Number of epithelial and pus cells were noted along with Gram reaction, morphology, and arrangement of the predominant organism. Respiratory samples were then inoculated on simple mannitol salt agar (MSA), blood agar, and chocolate agar at $37^{\circ} \mathrm{C}$ for 18-24 hours. Isolates were identified for M. catarrhalis on the basis of various biochemical tests, including gram staining, oxidase test, catalase test, and butyrate esterase production, and their inability to ferment sugars. Antibiotic susceptibility was determined by Kirby-Bauer disk diffusion method. Mueller-Hinton agar was used for susceptibility testing, and the results were concluded as the organism is either susceptible or resistant to antibiotics. As Pakistan is a developing country, cost is a big issue in health care. Quantitative cultures were not performed for the BAL or tracheal secretions. Also, no specific test was performed to assess beta-lactamase production in the isolates. To differentiate between actual pathogen and colonization infective markers, chest X-ray (CXR) changes and clinical assessment were also taken into account.

\section{Results}

There were total 110 patients with diagnosis of communityacquired lower respiratory tract infection. Out of them, 22 patients had culture positive for $M$. catarrhalis. Of these 22,14 were males and eight were females. The age distribution is listed in Table 1. The mean age of patients was 46.77 years with a range of $20-80$ years.

The isolates were 12 sputum samples, three BAL, and seven tracheal secretions.

Out of 22 patients, three patients presented with normal CXR and six patients had old changes in their CXR but had a history of productive cough and had positive infective markers. The remaining 13 patients had positive findings on their CXR. The CXR findings are shown in Figure 1.

Five patients were admitted in the intensive care unit (ICU), two patients in high dependency unit (HDU), and the rest of the 15 patients in ward.

Out of 22 patients, six patients had no known comorbidities and were between the ages of 25 years and 42 years and six patients only had asthma and presented with acute exacerbation with ages between 25 years and 55 years. There were three patients with the ages of 42 years, 62 years, and 68 years presenting acute exacerbation of chronic obstructive pulmonary disease (COPD), two patients previously diagnosed with interstitial lung disease, and one patient with lung fibrosis as shown in Figure 2. Other comorbidities of patients include diabetes, hypertension, and ischemic heart disease in one, five, and nine patients, respectively.

Regarding sensitivity patterns, $M$. catarrhalis was found to be sensitive to amoxicillin in 21 patients and ampicillin in eight patients as shown in Table 2, whereas it was $100 \%$ sensitive to ceftriaxone and $96 \%$ to cefuroxime. However, in terms of resistance patterns of $M$. catarrhalis, it was observed that it was resistant to ampicillin in 14 patients; clarithromycin in ten patients, out of which four patients were also resistant to erythromycin; and co-trimoxazole in 13 patients as shown in Table 2.

\section{Discussion}

Global increase in prevalence of $M$. catarrhalis has drawn our attention to this commensal of upper respiratory tract as

Table I Sex-wise age distribution among patients

\begin{tabular}{lllll}
\hline Sex & $20-40$ yrs & $41-60$ yrs & $61-80$ yrs & Total \\
\hline Male & 4 & 4 & 5 & 13 \\
Female & 4 & 4 & 1 & 9 \\
\hline
\end{tabular}




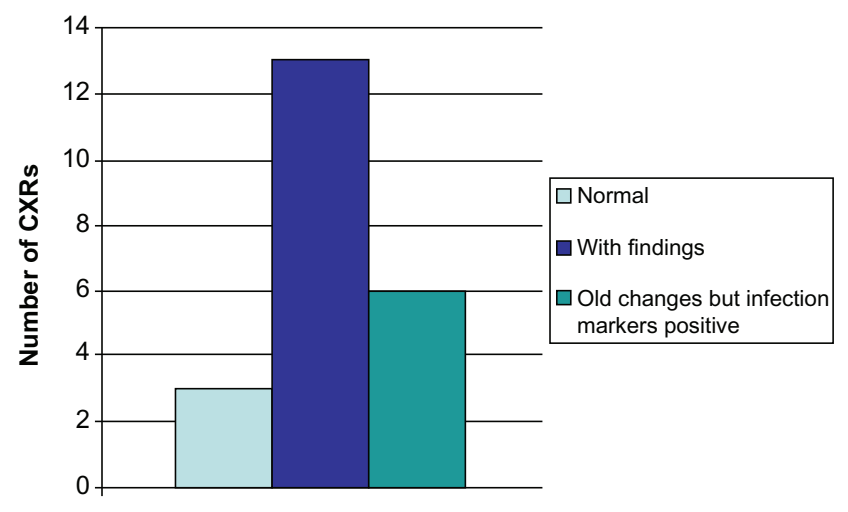

Figure I CXR findings.

Abbreviation: $C X R$, chest $X$-ray.

an important cause of lower respiratory infections for last 20-30 years. ${ }^{8}$ A study done in Pakistan by Abdullah et al in 2013 to evaluate the prevalence of $M$. catarrhalis in Karachi city showed that out of 776 sputum cultures from Essa laboratories, 39 were positive for $M$. catarrhalis, which is $5 \%$. Majority of the samples were from young individuals. ${ }^{9}$ This study was based on patients being treated as outpatients, whereas our study was only from the in-patient data of the chest department of a tertiary care hospital, where the prevalence is $20 \%$. This requires a larger study design to evaluate the true prevalence. This also defines that the majority of patients with $M$. catarrhalis infection require hospital admission, which can again be confirmed with a larger study design collecting data from all the hospitals in Karachi, Pakistan. Another study done in Rawalpindi, Pakistan, by Butt et al in 2005 showed $12 \%$ prevalence of $M$. catarrhalis in patients with pneumonia. ${ }^{10}$

Most important factor being considered is the increase in production of beta-lactamase in $M$. catarrhalis. Studies done in the United States and Canada reported beta-lactamase production in $M$. catarrhalis to be in excess of $90 \%,{ }^{11-13}$ thus explaining the increasing resistance. A European study done in 2002 showed that $98 \%$ of specimens produced

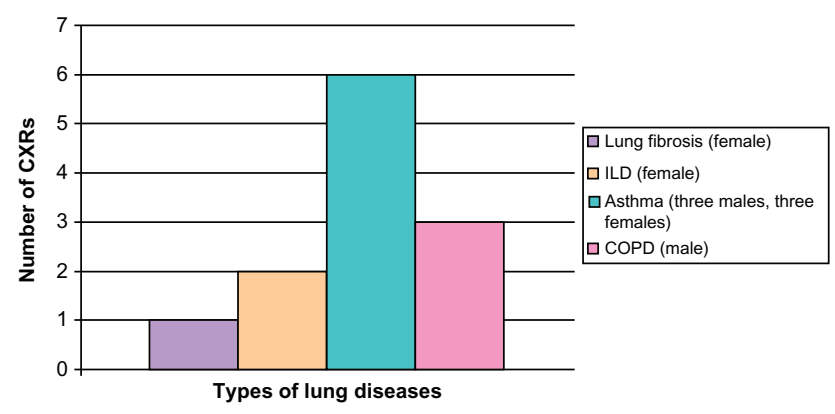

Figure 2 Distribution of lung etiologies in patients.

Abbreviations: CXRs, chest X-rays; ILD, interstitial lung disease; COPD, chronic obstructive pulmonary disease.
Table 2 Sensitivity and resistance pattern with resistance \%

\begin{tabular}{llll}
\hline Antibiotics & Sensitive & Resistant & $\begin{array}{l}\text { Total \% of } \\
\text { resistance }\end{array}$ \\
\hline Ampicillin & 8 & 14 & 63 \\
Amoxicillin-clavulanate & 21 & $\mathrm{I}$ & 4 \\
Ceftriaxone & 22 & 0 & 0 \\
Cefuroxime & 21 & $\mathrm{I}$ & 4 \\
Clarithromycin & 12 & 10 & 45 \\
Erythromycin & 18 & 4 & 18 \\
Co-trimoxazole & 9 & 13 & 59 \\
Levofloxacin & 9 & 13 & 59 \\
\hline
\end{tabular}

beta-lactamase. ${ }^{14}$ Most of the $M$. catarrhalis isolates in our study were resistant to ampicillin (63\%), presumably due to the increase in beta-lactamase production. A study done in a single hospital over a period of 10 years from 1984 to 1994 suggested that an increase in the minimum inhibitory concentration of antibiotics was not due to the increased frequency of beta-lactamase-producing strains but rather occurred mainly within the beta-lactamase positive strains. ${ }^{15}$ However, the resistance in our study was still low for amoxicillinclavulanate $(4 \%)$, ceftriaxone $(0 \%)$, and cefuroxime $(4 \%)$.

Recent studies also highlight the role of $M$. catarrhalis in the etiology of exacerbation of chronic respiratory diseases. ${ }^{16-18}$ In a study done in People's Republic of China, 7\% of patients with acute exacerbation of COPD had $M$. catarrhalis infection. ${ }^{19}$ Another study done by Domenech et al in Spain evaluating the infectious etiology of severe COPD patients found $M$. catarrhalis to be the cause in $15.4 \%{ }^{20}$ In our study, $13 \%$ of the patients presented with exacerbation of COPD, $31 \%$ with acute exacerbation of asthma in which $50 \%$ had a chronic history of asthma since childhood, $9 \%$ had interstitial lung disease, and only 4\% had lung fibrosis.

M. catarrhalis patterns of sensitivity and resistance need continuous surveillance. A study done in Taiwan to compare the data between 1993-1994 and 2001-2004 revealed an increase in minimum inhibitory concentration for cefaclor, cefuroxime, tetracycline, and co-trimoxazole, hence indicating increase in resistance over the years. ${ }^{21}$ A study done in Pakistan in 2002 by Tabassum and Ahmed determining the antibiotic susceptibilities showed $75 \%$ sensitivity to macrolides (clarithromycin and erythromycin) and 95\% sensitivity to quinolones (ciprofloxacin), ${ }^{22}$ whereas in our study sensitivity to clarithromycin and erythromycin has dropped to $45 \%$ and that for quinolone to $41 \%$. These differences also raise the need to conduct larger study for surveillance of the resistance pattern of $M$. catarrhalis in our region.

Another important factor to be considered is the pathogenicity of $M$. catarrhalis. In our study, $22 \%$ of patients required ICU admission and 9\% were admitted in HDU. By comparing 
our data with another study done in Karachi from a major laboratory based on outpatient population infected with $M$. catarrhalis, ${ }^{9}$ our study being done in a single department of a tertiary care hospital has similar numbers, we may conclude that most of the patient requires hospital admission. Less than $10 \%-30 \%$ of patients developing pneumonia with $M$. catarrhalis are believed to develop bacteremia, ${ }^{23}$ thus raising concerns about immunocompromised patients who develop lower respiratory infection with $M$. catarrhalis. Sugiyama et al reported a case of a 75-year-old female in Japan in 2000 who was immunocompromised due to agammaglobulinemia and was being treated at the hospital for acute pneumonia. Sputum culture grew M. catarrhalis as well as Pseudomonas aeruginosa; both were sensitive to imipenem, which was started immediately. Later, she developed infection of methicillin-resistant Staphylococcus aureus; despite continuous efforts, she failed to recover and died. On her autopsy, multiple lung abscesses of varying degrees were discovered indicating recurrent lung infections. ${ }^{24}$

Some studies suggest prescribing newer macrolides and quinolones as a second-line therapy for M. catarrhalis. ${ }^{25}$ Swanson et al compared the efficacy of 3 days treatment with azithromycin $500 \mathrm{mg}$ once daily with the 10 days treatment of clarithromycin $500 \mathrm{mg}$ twice daily in patients with chronic bronchitis, and found similar results. ${ }^{26}$ However, another study done on a smaller scale evaluating the pharmacokinetics and pharmacodynamics of moxifloxacin in community-acquired pneumonia concluded moxifloxacin to have excellent efficacy in treating patients with community-acquired pneumonia. ${ }^{27}$ But the resistance pattern of $M$. catarrhalis for these two drugs is yet to be evaluated.

In our study, we saw that the patients presenting with pneumonia were mostly males. Comorbidities in majority of patients were chronic respiratory illnesses. The prevalence of M. catarrhalis is increasing in our community. More patients infected with $M$. catarrhalis require hospital admission rather than outpatient treatment.

When we observe the sensitivity and resistance pattern, the highest resistance was already anticipated for ampicillin, but observing resistance with similar percentage to clarithromycin and levofloxacin is alarming. Sanford guide for antimicrobials suggests clarithromycin and flouroquinolones as a secondline drug for $M$. catarrhalis and clarithromycin as the first choice of therapy in patients with comorbidities presenting with community-acquired lower respiratory tract infection. ${ }^{28}$ Perhaps, we should consider newer macrolides (azithromycin) and quinolones (moxifloxacin) for the second-line treatment of $M$. catarrhalis, in view of increasing resistance patterns.
Keeping in mind the increasing prevalence of patients being infected with $M$. catarrhalis in our region, a larger scale research should be conducted to evaluate resistance pattern of $M$. catarrhalis, and with more definite results, we need to review the drug susceptibility in our region, hence, prescribing the more sensitive drug at an earlier phase of infection and improving the outcome of our patients.

\section{Acknowledgments}

Dr Safia Bader Uddin Shaikh would like to make the following statement: I am grateful to my parents and siblings who have always been there encouraging me through my toughest moments.

I would like to express my deepest appreciation to all those who provided me with their help and support to complete my research, especially my supervisor Dr Zafar Ahmed and my HOD (head of the department) Dr Syed Ali Arsalan. Also I thank Dr Aneel Chauhan for his guidance, and last but not the least, Mr Waqar Ahmed who would always make time in his busiest schedule. I would also like to thank the HOD of microbiology department Dr Saba Qaiser for letting me review the records.

\section{Disclosure}

The authors report no conflicts of interest in this work.

\section{References}

1. Jhonson MA, Drew WL, Roberts M. Branhamella (Neisseria) catarrhalis - a lower respiratory tract pathogen? J Clin Microbiol. 1981;13:1066-1069.

2. Aebi C. Moraxella catarrhalis - pathogen or commensal? Adv Exp Med Biol. 2011;697:107-116.

3. Brooks GF, Carroll KC, Butel JS, Morse SA, Mietzner TA. Chapter 16. Pseudomonas, acinetobacter, and uncommon gram-negative bacteria. Jawetz, Melnick, and Adelberg's Medical Microbiology. 26 ed. 2013. Available from: http://www.accessmedicine.com/content.aspx?a ID=57032597. Accessed November 17, 2013.

4. Sy MG, Robinson JL. Community-acquired Moraxella catarrhalis pneumonia in previously healthy children. Pediatr Pulmonol. 2010;45: 674-678.

5. Wallace RJ Jr, Steingrube VA, Nash DR, et al. BRO beta lactamases of Branhamella catarrhalis and Moraxella subgenus Moraxella, including evidence for chromosomal beta lactamases transfer by conjugation in $B$. catrarrhalis, M. nonliquefaciens, and M. lacunata. Antimicrob Agents Chemother. 1998;33:1845-1854.

6. Khan MA, Northwood JB, Levy F, et al. BRO beta lactamases and antibiotic resistances in a global cross sectional study of Moraxella catarrhalis from children and adults. J Antimicrob Chemother. 2010;65:91-97.

7. Wright PW, Wallace RJ Jr, Shepherd JR. A descriptive study of 42 cases of Branhamella catarrhalis pneumonia. Am J Med. 1990;88(5A):2S-8S.

8. Tamang MD, Dey S, Makaju RK, Jha BK, Shivananda PG, Bhramadatan KN. Prevalence of Moraxella catarrhalis infections of the lower respiratory tract in elderly patients. Kathmandu Univ Med J. 2005;3(9):39-44.

9. Abdullah FE, Ahjua KR, Kumar H. Prevalence and emerging resistance of Moraxella catarrhalis in lower respiratory tract infection in Karachi. J Pak Med Assoc. 2013;63(11):1342-1344. 
10. Butt T, Rafi N, Ahmed S, Ahmed RN, Salman M, Mirza SH. Community acquired pneumonia in Rawalpindi. PakJ Pathol. 2005;16: 6-14.

11. Doren GV, Jones RN, Pfaller MA, Kugler K, The Sentry Participant Group. Hemophilis influenza and Moraxella catarrhalis from patients with community acquired respiratory tract infections: antimicrobial susceptibility patterns from SENTRY antimicrobials surveillance program (United States and Canada,1997). Antimicrob Agents Chemother. 1999;43:385-389.

12. Jones RN, Jacobs MR, Washington JA, Pfaller MA. A 1994-1995 survey of Hemophilus influenza susceptibility to ten orally administered agents. A 187 clinical laboratory center sample in the United States. Diagn Microbiol Infect Dis. 1997;27:75-83.

13. Doren GV, Brueggemann AB, Pierce G, Holley HP, Rauch A. Prevalence of antimicrobial resistance among 723 out patient clinical isolates of Moraxella catarrhalis in the United States in 1994 and 1995: results of a 30 center national surveillance study. Antimicrob Agents Chemother. 1996;40:2884-2886.

14. Schmitz FJ, Beeck A, Perdikouli M, et al. Production of BRO beta lactamases and resistance to complement in European Moraxella isolates. J Clin Microbiol. 2002;40:1546-1548.

15. Walker ES, Preston RA, Post JC, Ehriic GD, Kalbfleisch JH, Kingman KL. Genetic diversity among strains of Moraxella catarrhalis: analysis using multiple DNA probes and a single locus PCR restriction fragment length polymorphism method. J Clin Microbiol. 1998;38: 1977-1983.

16. Hunter MH, King DE. COPD: management of acute exacerbations and chronic stable disease. Am Fam Physician. 2001;64(4):603-612.

17. Lieberman D, Lieberman D, Ben-Yaakov M, et al. Infectious etiologies in acute exacerbation of COPD. Diagn Microbiol Infect Dis. 2001;40(3): 95-102.

18. Sethi S, Murphy TF. Bacterial infection in chronic obstructive pulmonary disease in 2000: a state-of-the-art review. Clin Microbiol Rev. 2001;14(2):336-363.
19. YE F, He LX, Cai BQ, et al. Spectrum and antimicrobial resistance of common pathogenic bacteria isolated from patients with acute exacerbation of chronic obstructive pulmonary disease in mainland of China. Chin Med J. 2013;126(12):2207-2214.

20. Domenech A, Puig C, Martí S, et al. Infectious etiology of acute exacerbations in severe COPD patients. $J$ Infect. 2013;67:516-523.

21. Hsu SF, Lin YT, Chen TL, et al. Antimicrobial resistance of Moraxella catarrhalis isolates in Taiwan. J Microbiol Immunol Infect. 2012;45: 134-140.

22. Tabassum N, Ahmed A. Determination of anti-microbial susceptibilities of H. Influenza, S. pneumonia and M. catarrhalis. J Pak Med Assoc. 2002;52:87-90.

23. Levison ME. Pneumonia including necrotizing pulmonary infections (lung abscess). In: Fauci AS, Braunwald E, Isselbacher KJ, Wilson JD, Martin JB, Kasper DL, et al, editors. Harrison's Principles of Internal Medicine. 14th ed. New York: Mc Graw Hill; 1998:1437-1445.

24. Sugiyama H, Ogata E, Shimamoto Y, et al. Moraxella catarrhalis pneumonia in a patient with immunoglobulin deficiency. J Infect Chemother. 2000;6:61-62.

25. Bandak SI, Turnak MR, Allen BS, et al. Antibiotic susceptibilities among recent clinical isolates of $H$. influenzae and Moraxella catarrhalis from fifteen countries. Eur J Clin Microbiol Infect Dis. 2001;20:55-60.

26. Swanson RN, Lainez-Ventosilla A, De Salvo MC, Dunne MW, Amsden GW. Once daily azithromycin for 3 days compared with clarithromycin for 10 days for acute exacerbation of chronic bronchitis: multicenter, double-blind, randomized study. Treat Respir Med. 2005; 4(1):31-39.

27. Yoshida K, Okimoto N, Kishimoto M, et al. Efficacy and safety of moxifloxacin for community acquired bacterial pneumonia based on pharmacokinetic analysis. J Infect Chemother. 2011;17(5):678-685.

28. Gilbert DN, Moellering RC Jr, Chambers HF, Saag MS. The Sanford Guide to Antimicrobial Therapy. 43 ed. Sperryville: Antimicrobial Therapy Inc; 2013.
Infection and Drug Resistance

\section{Publish your work in this journal}

Infection and Drug Resistance is an international, peer-reviewed openaccess journal that focuses on the optimal treatment of infection (bacterial, fungal and viral) and the development and institution of preventive strategies to minimize the development and spread of resistance. The journal is specifically concerned with the epidemiology of antibiotic

\section{Dovepress}

resistance and the mechanisms of resistance development and diffusion in both hospitals and the community. The manuscript management system is completely online and includes a very quick and fair peerreview system, which is all easy to use. Visit http://www.dovepress.com/ testimonials.php to read real quotes from published authors. 